\title{
Precipitación acumulada en las tierras altas entre las secciones Tilarán y Central, Costa Rica
}

\author{
Accumulated precipitation in high land between the Tilaran and \\ Central sections, Costa Rica
}

Marvin E. Quesada Q., Departamento de Ciencias Sociales, Universidad de Costa Rica, Costa Rica, marvin.quesada@ucr.ac.cr

\begin{abstract}
Resumen: Se analiza el total acumulado de la precipitación cada cinco año, utilizando tres estaciones meteorológicas: Zarcero, Piedades Sur y Montes de Oro en Costa Rica. Estas localidades fueron seleccionadas, ya que cada una se ubica en sectores con distintos niveles de precipitación. De esta manera es posible tener una idea del comportamiento de la precipitación a lo largo de veinte años. Esto debido a que, por ser estaciones ubicadas en sectores muy rurales, solo una de ellas, la primera tiene datos superiores a los cincuenta años, pero para homogenizar los registros se tuvo que considerar solo veinte años. Los periodos históricos cada cinco años secos o húmedos se identifican mediante el método de índice de precipitación estandarizado y sus tendencias se analizan para evaluar la posible relación con el evento ENOS. Todas las estaciones evidencian cierta igualdad en la distribución quinquenal en la precipitación en el transcurso del período de estudio (1994-2013). La tendencia en cada estación es la siguiente, Zarcero, muestran niveles altos en el periodo 2004-2008. En Montes de Oro los niveles de precipitación son muy regulares, aunque con un ligero incremento entre el 20042008, pero en realidad es muy leve el incremento. En el caso de Piedades Sur se muestra un fuerte incremento en la lluvia en el quinquenio de 2004-2008. Esto refleja la existencia de una relación entre la precipitación del área en estudio con respecto a evento del ENOS, así como la influencia de la irregularidad en el relieve montañoso.
\end{abstract}

Palabras clave: Precipitación; climatología; quinquénios; sectores montañosos; ENOS.

\begin{abstract}
It is analyzed a cumulative total of precipitation every five years, using three climatological stations: Zarcero, Piedades Sur and Montes de Oro in Costa Rica. These locations are selected, since each one is located in different areas of precipitation. In this way it is possible to get an idea of the behavior of precipitation over twenty years. Historical periods every five dry or wet years are identified by the standardized precipitation index method and their trends are analyzed to assess the possible relationship with the ENSO event. Because, those stations are located in very rural areas, only one of them, the first has more than fifty-year data, but to homogenize the records had to consider only twenty years. All stations show some equality in the five-year distribution in precipitation over the course of the period of study (1994-2013). The trend in each season is as follows, Zarcero, they show high levels in the period 2004-2008. Montes de Oro precipitation levels are very regular, albeit with a slight increase in between 2004-2008, but in reality, the increase is very mild. In the case of Piedades Sur shows a strong increase in the rain on the five-year period from 2004-2008. This reflects the existence of a relationship between the area precipitation study regarding ENSO event.

As well as the mountainous irregularity.
\end{abstract}

Keywords: Precipitation; climatology; five-year periods; mountainous areas; ENSO.

\section{Introducción}

El conocimiento y análisis de la acumulación de la precipitación cada cinco años en un área o región durante algunos años consecutivos, resulta de vital importancia para 
visualizar la tendencia en los patrones de precipitación, si estos tienden a ser húmedos o secos. El conocimiento de la acumulación de las lluvias en sectores montañosos y en sus faldas, aparte de que ha sido poco estudiada, es muy variable dadas las condiciones particulares que poseen los sectores montañosos. Muchas veces se comete el error en generalizar que una región posee un nivel de precipitación homogéneo, solo porque se ubica en una región climática reconocida mundialmente.

Sin embargo, no se consideran factores como la latitud, la altitud, la exposición, el eje montañoso, entre otros. Similarmente, existen otros eventos como el ENOS (EI NiñoLa Niña- Oscilación del Sur), así como factores de circulación general como masas de aire, vientos, centros de alta y baja presión que modifican en cortas distancias los niveles de precipitación, dadas las diferencias en las masas de aire o por la canalización de vientos, entre otros.

Para hacer un estudio climático es importante contar con suficiente cantidad de datos, mientras más años se cuenten para hacer un estudio, se tendrán resultados más confiables. En los países con escasos recursos, por lo general, cuentan con escasos registros climáticos. tanto en cantidad como en calidad. Costa Rica cuenta con registros superiores a veinte años en varias estaciones ubicadas en la región central y en los principales puertos o aeropuertos. Sin embargo, existen otros sectores como son las áreas rurales, y los macizos montañosos que cuentan con escasas estaciones y poca cantidad de años de registro.

En este caso se investigará las variaciones de los totales anuales de precipitación, en periodos de cinco años, en los sectores montañosos entre las secciones de Tilarán y Central en Costa Rica. Esta región muestra variaciones que se debe sobre todo a factores como el relieve, la altitud y por elementos como el viento, la nubosidad. Por ser un sector con un relieve irregular se vuelve aún más inestable la variación espacial de la precipitación, al convertirse el factor orográfico un elemento primordial en la formación de microclimas. Otro factor imperativo y que hace de esta investigación aún más atractiva, es que el sector bajo estudio, abarca dos vertientes muy distintas en su régimen de precipitación que son: la vertiente Pacífica y la subvertiente Norte.

Como ya se mencionó el factor orográfico, la influencia de ciertos vientos, ondas tropicales, frentes fríos, entre otros, permiten la existencia de variadas precipitaciones. Con esto, se trata de explicar que en estos sectores montañosos son típicas las lluvias intensas y de corta duración o de poca precipitación, pero de muy larga duración. Estas pueden variar al mismo tiempo en cortas distancias por razones como son las depresiones, serranías, cerros y los cañones de los ríos, entre otros. Estos parámetros hacen indispensable analizar los totales de precipitación cada cinco años con la idea de verificar si existen diferencias en los niveles de precipitación, tanto por cada cinco años como en el lugar estudiado (Figura 1). 


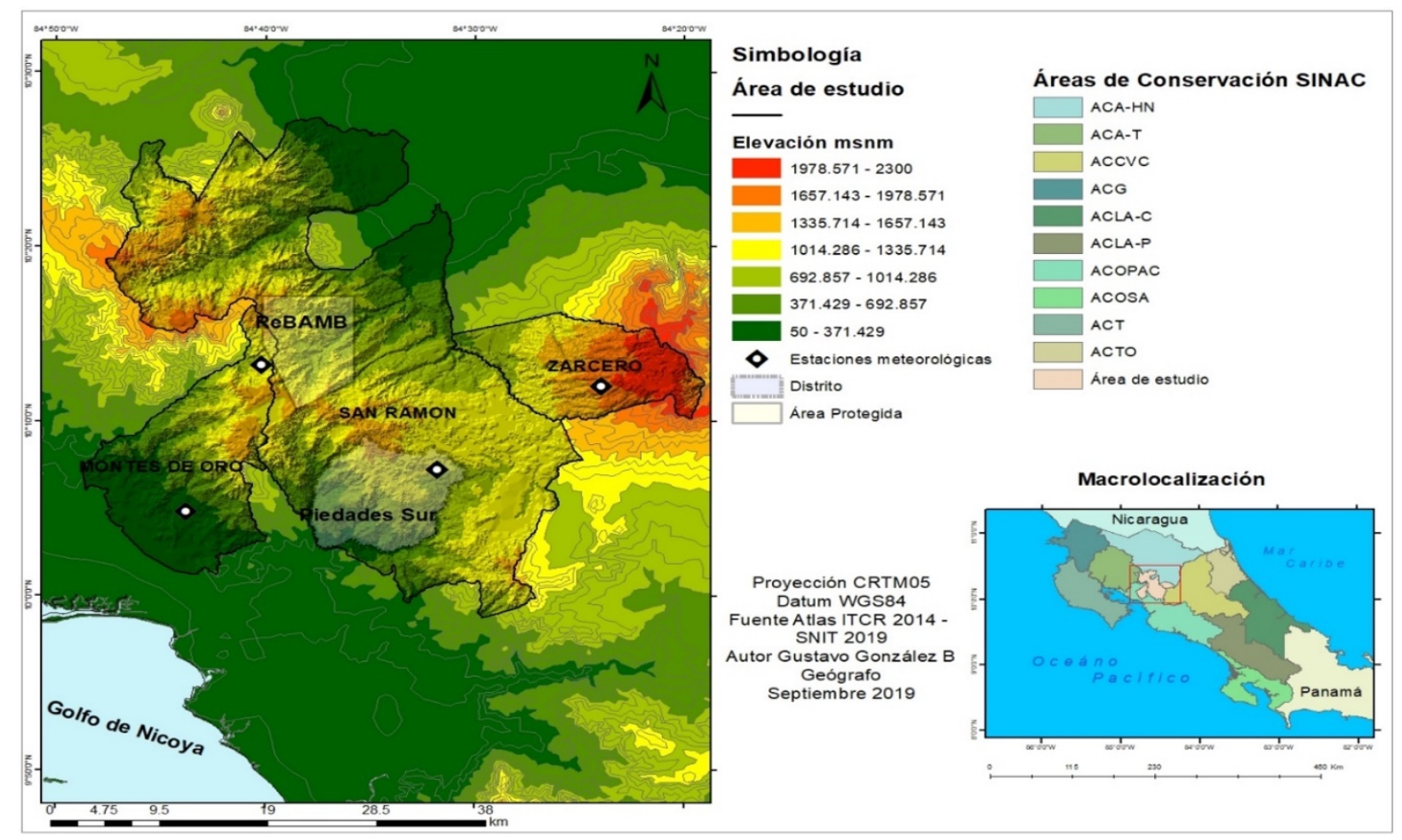

Figura 1: Ubicación del sector montañoso y de pie de monte del sector en estudio.

Fuente: Atlas ITCR (2014) y SNIT (2019).

\section{Mecanismos físicos de la precipitación}

Los mecanismos físicos de la precipitación anual en los sectores ubicados entre las secciones de Tilarán y Central ocurren de dos formas: en el sector de la subvertiente norte, las precipitaciones son abundantes durante todo el año. En tanto, en el sector Pacífico se producen dos periodos bien definidos: uno que va de mayo a octubre y otro de diciembre a marzo con dos meses de transición que son abril y noviembre. Los grandes sistemas meteorológicos y sinópticos que modulan las precipitaciones de mayo a octubre están ligados al funcionamiento de la Zona de Convergencia Intertropical (ZCIT) (Hastenrath, 2002), los vientos Oestes Ecuatoriales que tienen su mayor fuerza durante los meses citados. En el período Mayo-Junio y SeptiembreOctubre (MJ y SO), ocurren las épocas con mayor precipitación en la vertiente Pacífica. Mientras que en Julio-Agosto (JA) está modulada por la migración hacia el Norte de la Zona de Convergencia Intertropical (Moura y Shukla, 1981; Nobre y Shukla, 1996; Hastenrath, 2002).

La variabilidad de la precipitación en la subvertiente Norte está ligada a la variabilidad del flujo Alisio del Noreste, que influye especialmente sobre la vertiente Caribe durante gran parte del año. Además, es frecuente la aparición de ondas tropicales. Estos vientos proveen el aire tropical húmedo-cálido para activar la convección y precipitación en las llanuras y partes altas de Costa Rica.

Los vientos fríos son fuertes y también llegan hasta los sectores altos. Estos son conocidos como Los "Nortes" son vientos que soplan violentamente por varios días. Se originan por la invasión de masas de aire polar continental modificado, procedentes 
del norte de los Estados Unidos y sur de Canadá. Estos vientos al pasar sobre el Golfo de México recogen abundante humedad que después es liberada en forma de lluvia.

En tanto, las ondas tropicales tienen la característica de que aparecen entre los meses de mayo y octubre y se originan como producto de los disturbios atmosféricos. Estos normalmente inducen a lluvias lentas y de larga duración a cualquier hora del día o la noche.

Ambos factores, tanto los provenientes de Norte, Noreste y Este de América como del Oeste de Suramérica están modulados por la oscilación del sur y por "El Niño o La Niña" en la escala anual (Zhou y Lau, 2001).

El llamado evento de EI Niño-Oscilación del Sur (ENOS) es considerada una de las fuentes predominantes en la variabilidad del clima alrededor del mundo (Trenberth y Caron, 2000). EI ENOS, es un fenómeno de escala planetaria relacionado con la importante y compleja interacción océano-atmósfera sobre el Pacífico Tropical Ecuatorial, presentándose un ciclo de "El Niño" (fase cálida) y la "La Niña" (fase fría) en la opuesta. Las características principales en la atmósfera y el océano asociadas a los episodios de "ENOS" son: predominancia de anomalías positivas en la temperatura de la superficie del mar (SST, siglas en inglés), debilitamiento de los vientos del Este en superficie, baja presión con convección profunda en el Pacífico oriental y alta presión con movimientos de subsidencia en el Pacífico oeste, Indonesia y Australia. En los eventos "La Niña" generalmente se revierten los patrones en la atmósfera y el océano (Kousky y Ropelewski, 1989). Estos patrones anómalos ocurren sobre la cuenca del Pacífico tropical, incluyendo una extensa área sobre los trópicos (más de un tercio del cinturón tropical alrededor del globo).

Por consiguiente, en esta investigación se pretende analizar los acumulados totales anuales cada cinco años, utilizando el registro histórico de tres estaciones climáticas. Es conocido por ciertos científicos como (Waylen and Quesada, 2001) que pareciera que existe un factor isostático entre ambos océanos, cuando el Pacífico Ecuatorial Tropical se manifiestan TSM inferiores al promedio, en el océano Atlántico Tropical Norte se manifiestan TSM superiores al promedio. Esta condición atmosférica podría influir en la merma de las lluvias de ambas vertientes. Es imperativo averiguar la acumulación anual de precipitación en las tres estaciones bajo estudio.

En vista de que son variados los patrones que inducen a la precipitación en el área en estudio, es imperativo hacer un análisis acumulado en periodos de cada cinco años, donde se tiene una estación del sector Subvertiente Norte y dos del sector Pacífico (Tabla I). 
Tabla I: Totales acumulados de precipitación por estación en veinte años.

\begin{tabular}{|l|c|}
\hline Estación & $\begin{array}{c}\text { Totales precipitación en veinte años } \\
\text { Climatológica }\end{array}$ \\
\hline Zarcero & 41171,8 \\
\hline Piedades Sur & 58553,9 \\
\hline Montes de Oro & 63256,2 \\
\hline
\end{tabular}

Fuente: IMN (2017).

\section{Ubicación}

El área de estudio se ubica en parte en el sector cercano a la línea divisoria de aguas y el otro en las faldas de dichos sectores montañosos de Costa Rica (Figura 1). Dichos sectores se convierten asimismo en dos zonas distintas: Una parte presenta una única estación la lluviosa, y otra con dos periodos: el lluvioso y el seco.

Las estaciones climatológicas en estudio se encuentran localizadas entre dos sistemas montañoso conocido como secciones de Tilarán y Central. Cada una se encuentra a una distancia de aproximadamente de veinte kilómetros. Las estaciones utilizadas son Zarcero ubicada en el sector de la subvertiente Norte muy cerca de la divisoria continental, las estaciones de Piedades sur y Montes de Oro, ambas en el sector Pacífico.

\section{Métodos}

Para el estudio del clima resulta imprescindible realizar análisis de datos que se colectan diariamente, con el objetivo de calcular variaciones a largo plazo (quinquenios). Para la realización de esta investigación se consideraron tres estaciones, Los datos climatológicos de precipitación fueron suministrados por el Instituto Meteorológico Nacional de Costa Rica.

En la prueba Mann-Kendall (Mann 1945; Kendall 1975) los datos se evalúan como una serie temporal ordenada. Cada serie se divide en periodos de cinco años (quinquenio). Se suman las series anuales y se obtiene el porcentaje de cambio. De esta forma, se gráfica la serie y se obtienen los cambios que ha sufrido toda la serie temporal en periodos húmedos y secos o relativamente secos por ser sectores montañosos lluviosos. Se supone que el valor inicial de la estadística Mann-Kendall, $\mathrm{S}$, es 0 (por ejemplo, sin tendencia).

Si un dato de un período de tiempo posterior es mayor que un dato de un período de tiempo anterior, S se incrementa en 1. Por otro lado, si los datos de un período de tiempo posterior son inferiores a los de los datos muestreados anteriormente, $\mathrm{S}$ se reduce en 1.

El índice de precipitación estandarizado (Mckee et al., 1993) es un índice de comparación de las series quinquenales, que está basado en la probabilidad de precipitación para varios periodos de tiempo, por ejemplo, uno, dos, tres, cuatro y más periodos de cinco años. Esta proporciona una comparación de la precipitación durante 
un período específico con los totales de precipitación del mismo período para todos los años incluidos en el registro histórico. En consecuencia, facilita el análisis temporal de los fenómenos húmedos y secos. Los datos históricos de lluvia de cada estación se ajustan a una probabilidad gamma

La serie temporal por quinquenios se correlacionó con las teleconexiones de los SOI, con base en los registros del Centro de Predicción Climática, la Administración Nacional Oceánica y Atmosférica (NOAA) (http://www.cpc.ncep.noaa.gov/ data/índices/soi), Estados Unidos.

\section{Resultados}

En la realización del análisis a nivel quinquenal se tiene que las tres estaciones muestran ligeras diferencias. Por lo tanto, se hace una descripción de su comportamiento climático de la precipitación. Los siguientes análisis son comparados con la tabla de la NOAA sobre las series mensuales de los eventos del ENOS. La estación de Zarcero (Tabla II; Figura 2) muestra que el quinquenio de 1999- 2003 es de 10217,4 mm, con los datos más bajos de todo el registro histórico. Sin embargo, para el quinquenio de 2004-2008 se tienen datos de 11113,6 mm, existiendo una mayor cantidad de precipitación. Se muestra claramente la influencia de eventos extremos como el ENOS.

Tabla II: Precipitación total acumulada quinquenal $(\mathrm{mm})$ en la estación meteorológica de Zarcero Años Precipitación Zarcero $(\mathrm{mm})$

\begin{tabular}{|l|l|}
\hline $1994-1998$ & 10503,5 \\
\hline $1999-2003$ & 10217,4 \\
\hline $2004-2008$ & 11113,6 \\
\hline $2009-2013$ & 10278,3 \\
\hline
\end{tabular}

Fuente: IMN (2017).

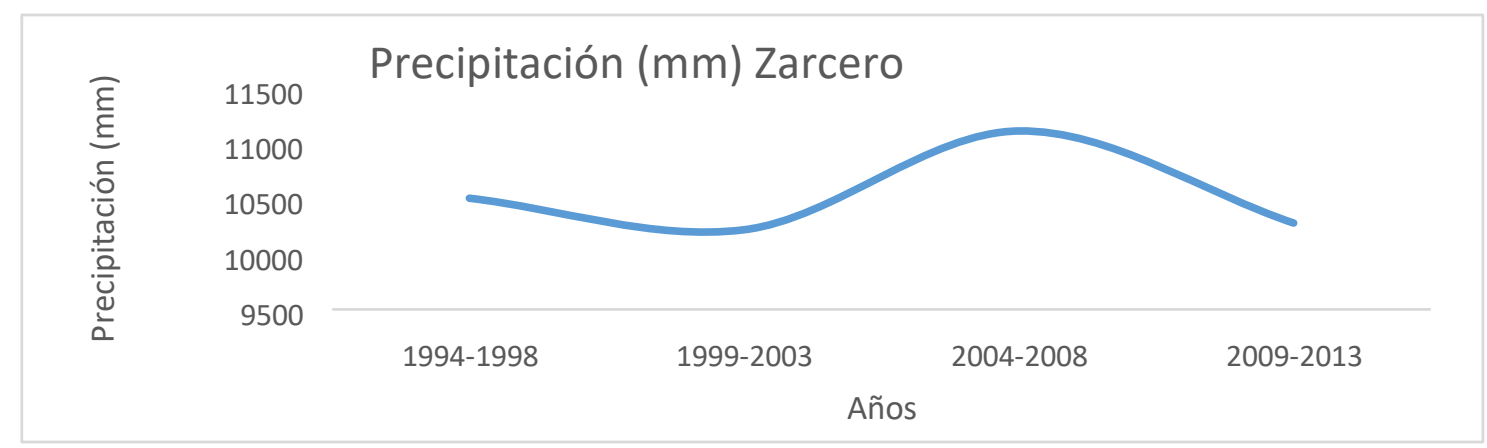

Figura 2: Precipitación quinquenal $(\mathrm{mm})$ en Zarcero.

Fuente: IMN (2017).

Para la estación climatológica de Piedades Sur (Tabla III) se nota que el quinquenio que registró sus valores más bajos es 1994-1998 mm. Mientras que para el quinquenio 
de 2004-2008 se tienen altos valores de precipitación, 23470,20 mm. Que es precisamente a nivel quinquenal la estación que muestra los valores más altos durante todo este estudio. Sin embargo, es muy interesante anotar que el quinquenio de 19941998, que mostró ser una de las zonas con mayor disminución en toda el área en estudio. Esta disminución es consistente con la variabilidad propia de la precipitación, considerando que puede haber otros factores que influyan en su comportamiento, como podría ser el impacto o persistencia de años ENOS o la incursión de las masas de aire provenientes del sector de la Subvertiente Norte a través del paso intermontano de Tapezco (Figura 3).

Tabla III: Precipitación quinquenal $(\mathrm{mm})$ en la estación meteorológica de Piedades Sur.

\begin{tabular}{|l|c|}
\hline Años & \begin{tabular}{c} 
Precipitación Piedades Sur (mm) \\
\hline $1994-1998$
\end{tabular} \\
\hline $1999-2003$ & 1389,80 \\
\hline $2004-2008$ & 23470,20 \\
\hline $2009-2013$ & 15375,80 \\
\hline
\end{tabular}

Fuente: IMN (2017).

Para el quinquenio de 1994-1998, se mantienen los menores valores de la precipitación. Para el periodo 1999-2003, se registran ligeramente superiores, hasta llegar a tener los datos superiores en el quinquenio de 2004-2008, luego de nuevo se produce un ligero descenso (Figura 3).

Para el quinquenio de 2004-2008 el incremento de la precipitación quinquenal muestra mayores datos, los cuales son contrastables con respecto al quinquenio de periodo 1994-1998. Esto demuestra una fuerte influencia de la fase fría del ENOS, dado que se presentaron muchas precipitaciones. Esta región muestra una alta variabilidad e impacto del ENOS en periodos como el 1994-1998, 1999-2003 y en 2004 y 2008 . Por supuesto bajo diferentes circunstancias.

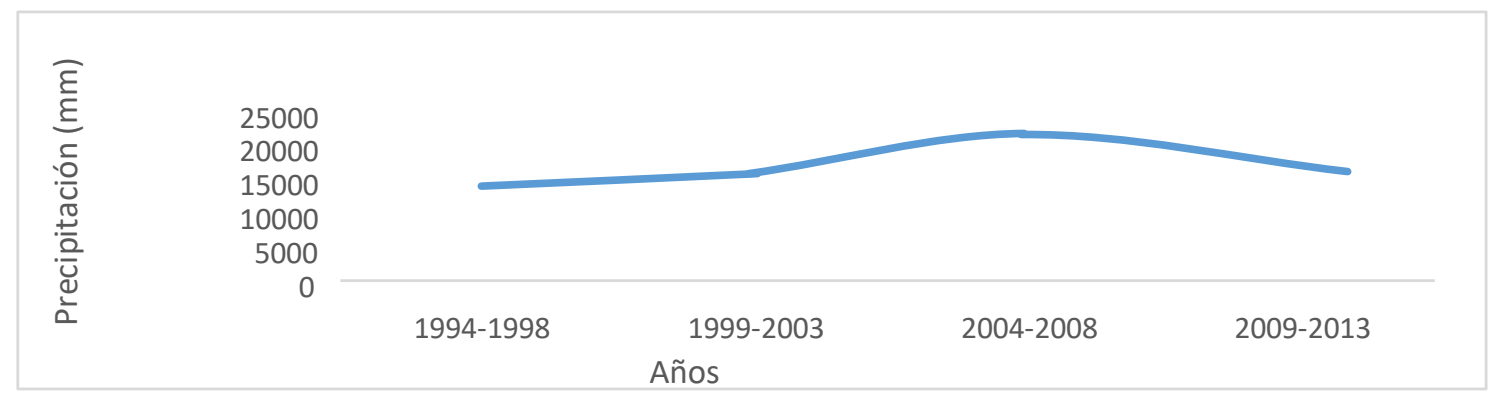

Figura 3: Precipitación quinquenal de Piedades Sur (mm).

Fuente: IMN (2016). 
La estación de Montes de Oro (Tabla IV; Figura 4) tiene la particularidad mostrar un comportamiento muy homogéneo, lo cual se debe a su posición geográfica en la parte alta de un cerro, muestra constantes precipitaciones durante la mayor parte del día.

Tabla IV: Precipitación quinquenal (mm) en la estación meteorológica de Montes de Oro.

\begin{tabular}{|l|r|}
\hline Años & Precipitación Montes de Oro \\
\hline $1994-1998$ & 15432,42 \\
\hline $1999-2003$ & 17290,20 \\
\hline $2004-2008$ & 19286,70 \\
\hline $2009-2013$ & 15610,60 \\
\hline
\end{tabular}

Fuente: IMN (2017).

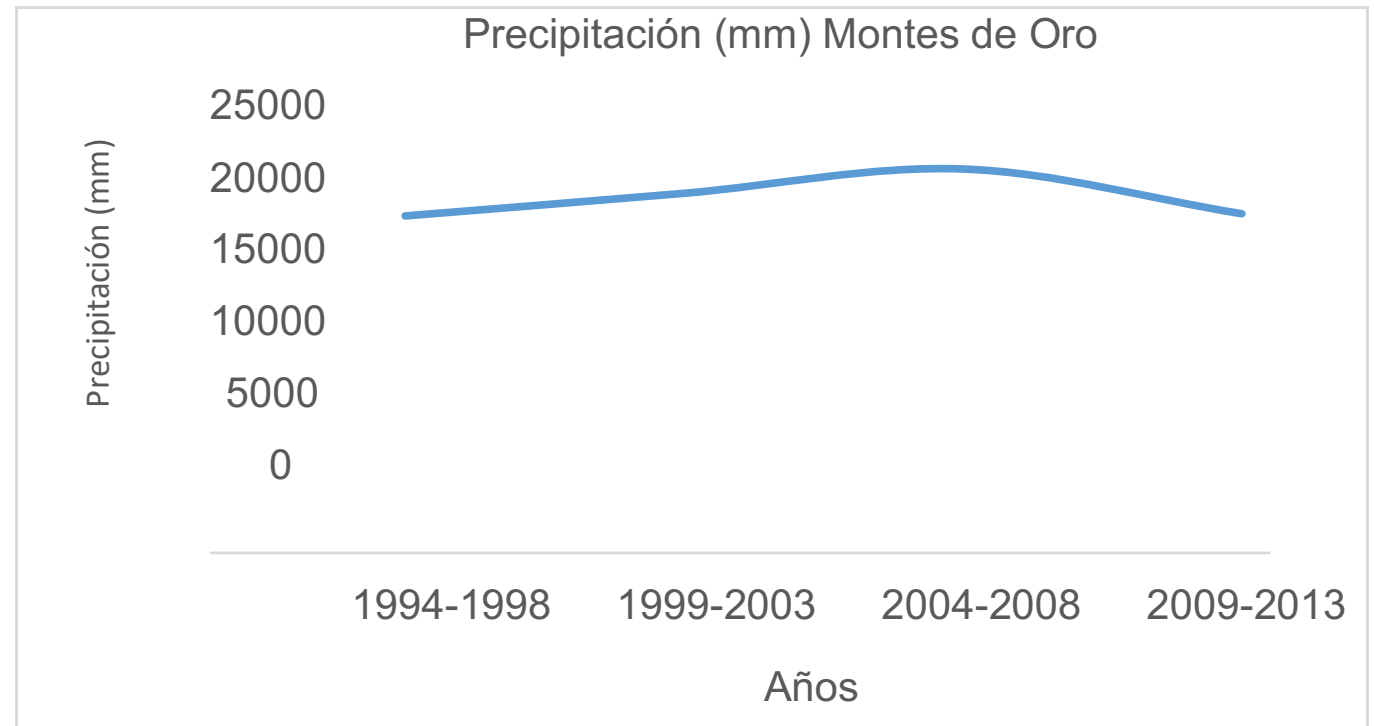

Figura 4: Precipitación quinquenal en Montes de Oro.

Fuente: IMN (2017).

A pesar de que estas dos últimas estaciones pertenecen a una misma vertiente presentan características muy distintas. La primera, Piedades Sur, tiene la misma tendencia que muestra una estación típica de la vertiente Pacífica. En tanto, Montes de Oro tiene una particularidad única en este caso de presentar un comportamiento muy homogéneo, pareciera que no existe influencia de ninguna de las dos fases del ENOS.

Algunos científicos tales como Enfield y Alfaro (1999), Giannini et al. (2000) y Quesada y Waylen (2008) han considerado que las anomalías de las temperaturas superficiales del océano Atlántico Tropical Norte y el Pacífico Ecuatorial en la zona del El Niño 3.4 que se ubica en el océano Pacífico Ecuatorial definitivamente influyen en la distribución interanual de la precipitación. De ahí que se cada cinco años, se noten diferencias en los promedios de lluvia, incluso con diferencias muy marcadas. El quinquenio de 1999-2003 está íntimamente relacionado con el efecto de El Niño. Por su ubicación en la vertiente Pacífico es típico la reducción de las precipitaciones. En 
cambio, en el quinquenio del 2004-2008 se presenta la fase fría del ENOS. Durante este periodo se dio la fase fría del ENOS, donde se nota un incremento en las precipitaciones, En tanto, en el sector Subvertiente Norte y Caribe más bien se dio un aumento de las precipitaciones entre junio del 2004 hasta Enero del 2007.

La estación de Zarcero (Figura 5) es la estación que muestra los niveles mínimos de precipitación, dado a que se ubica por encima del óptimo pluviométrico. Cuando una localidad se ubica por encima de altitudes cercanas a los 1700 m.s.n.m. existe una regularidad en los niveles de precipitación. Esto se debe a que estas altitudes las lluvias disminuyen y la influencia del evento ENOS es típico de la vertiente Pacífica.

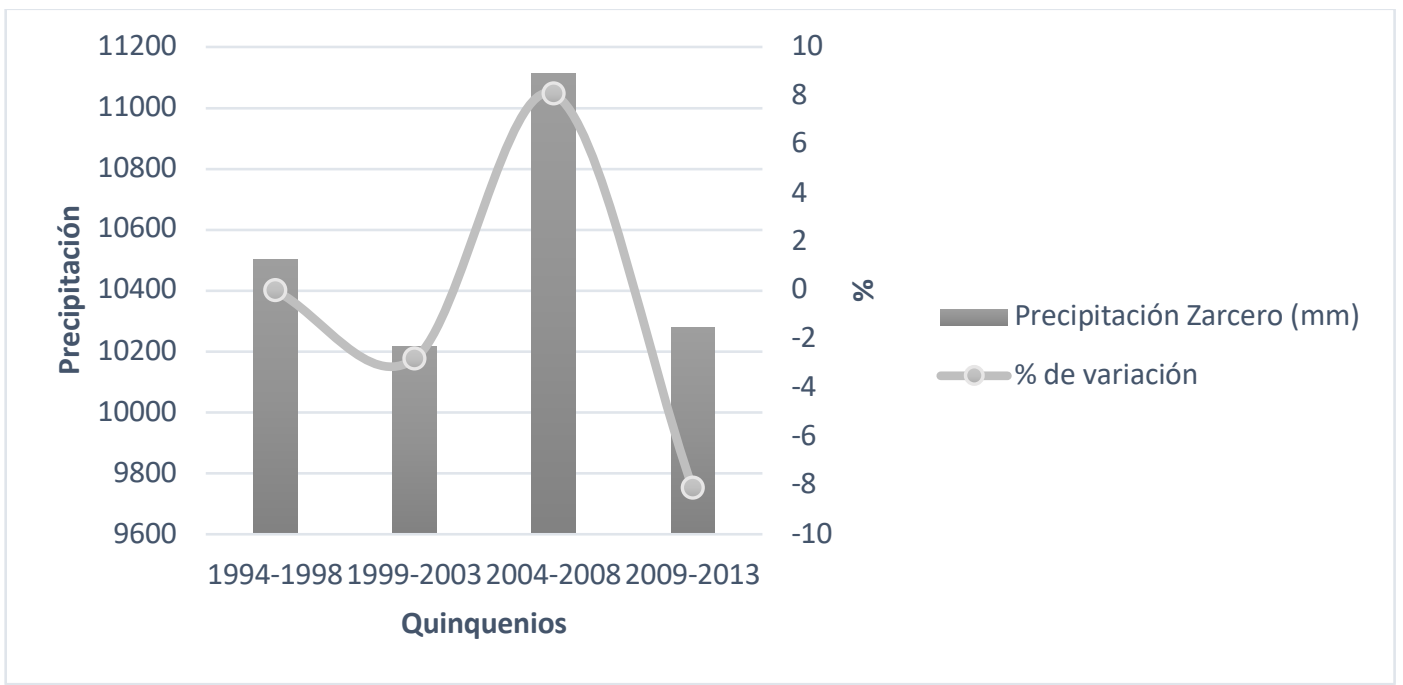

Figura 5: Variación porcentual de la precipitación anual acumulada en Zarcero.

Fuente: IMN (2017).

Mientras tanto, en la estación de Piedades Sur (Figura 6) durante la fase cálida del ENOS, en Agosto de 1994, los niveles son bajos. No obstante, a partir de ese momento inicia un ascenso constante hasta el 2004. Sin embargo, durante la fase fría del ENOS se encontró que desde Octubre de 2005 hasta Mayo de 2008 se dieron intensas precipitaciones, incluso de las estaciones en análisis, siendo precisamente la estación climática que muestra los mayores niveles de precipitación. 


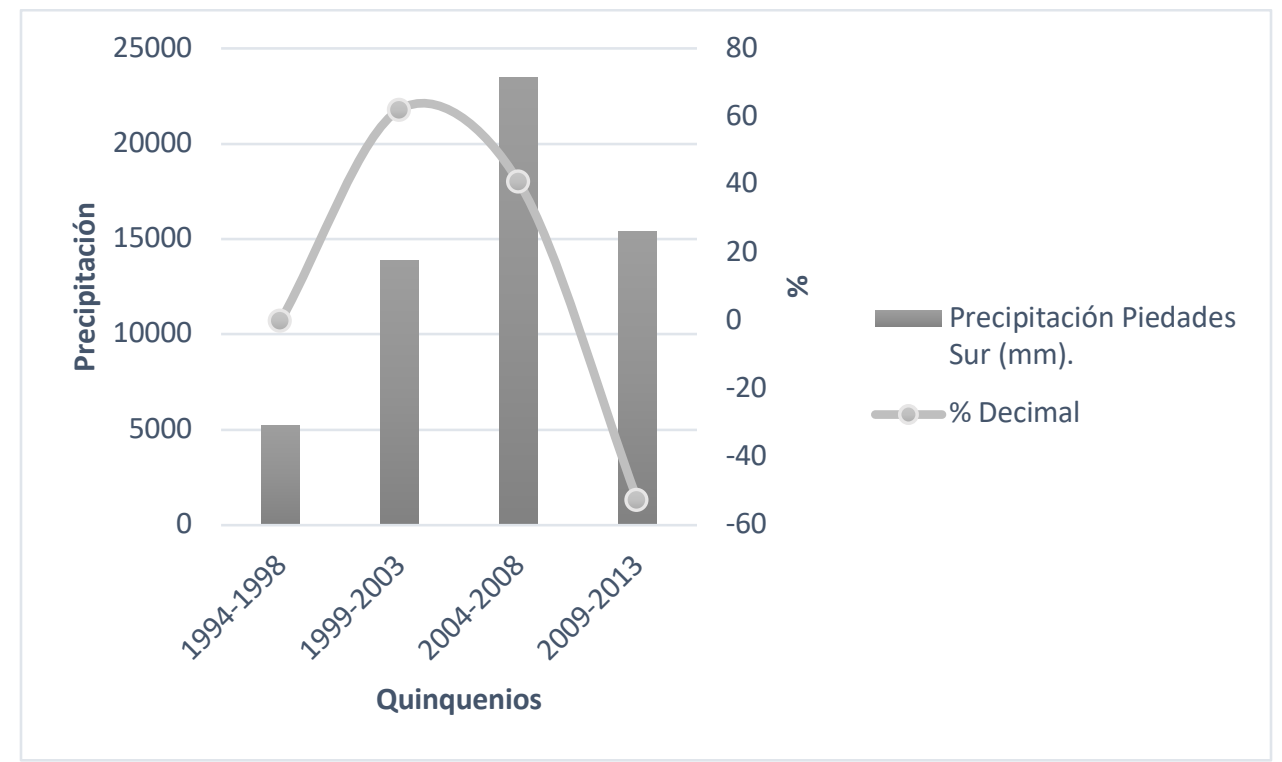

Figura 6: Variación porcentual de la precipitación anual acumulada en Piedades Sur.

Fuente: IMN (2017).

En el caso de la estación de Montes de Oro (Figura 7) se tiene un comportamiento muy regular, dada su ubicación cerca en la parte más alta del macizo. Existe un aumento lluvias desde Octubre del 2004 hasta Mayo de 2008, lo cual se debe a la mayor influencia del evento de La Niña en la vertiente Pacífica.

La estación de Montes de Oro a pesar de estar ubicada en sector montañoso con una persistente precipitación durante gran parte del día, Los dos quinquenios que muestran menor niveles de precipitación son 1994-1998 y 2009-2013, aunque no es muy marcada. En tanto, el resto de la serie bajo estudio tiene una correspondencia directa con la fase Fría del ENOS.

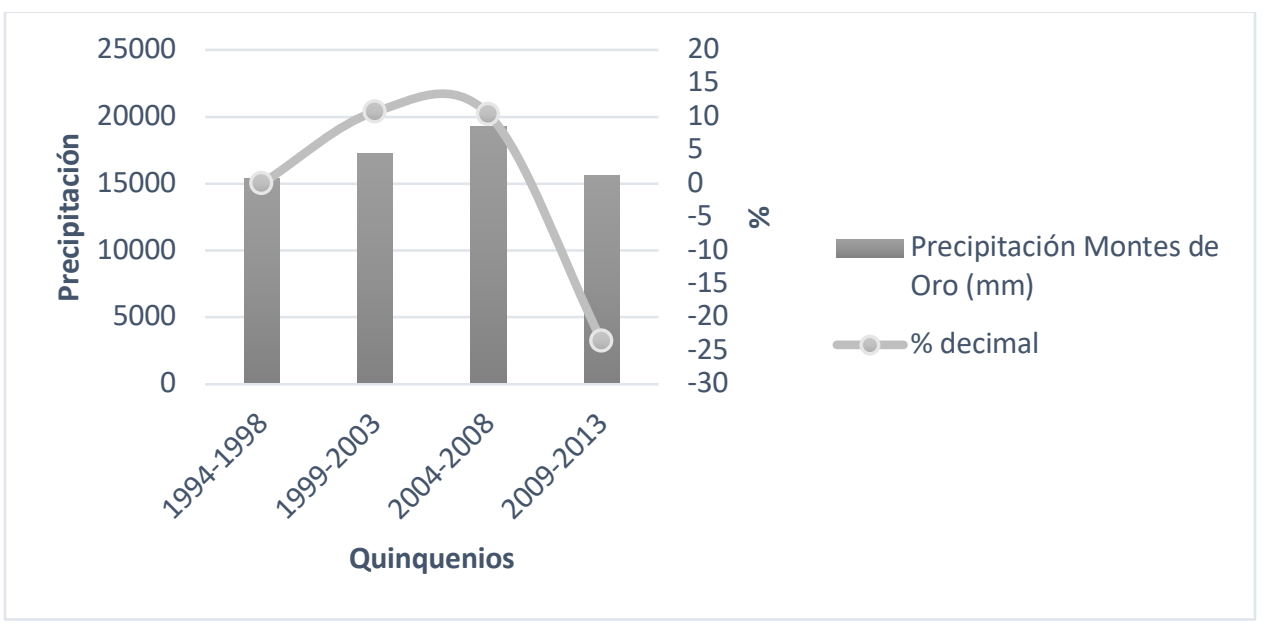

Figura 7: Variación porcentual de la precipitación anual acumulada en Montes de Oro.

Fuente: IMN (2017). 
En el caso de la estación meteorológica de Piedades Sur se nota una situación muy similar, lo cual se debe a su ubicación en la vertiente Pacífica. Los quinquenios de 1994-1998 y 2009-2013, muestran un descenso considerable en las lluvias que coincide con El Niño. Mientras tanto, en los quinquenios intermedios entre estos periodos se presentan niveles de precipitación muy superiores al promedio. Estos niveles de precipitación coinciden con el evento La Niña.

Entre las causas que podrían provocar las fluctuaciones quinquenales, están influencia de patrones de circulación a nivel estacional. Además, las diferencias de patrones de temperaturas superficiales de las aguas de los océanos Atlántico Tropical Norte y el Pacífico Ecuatorial. Es imperativo también las distintas altitudes y las diferencias de relieve a las que se encuentran las tres estaciones en estudio (Nobre and Shukla,1996; Durand et al., 2010).

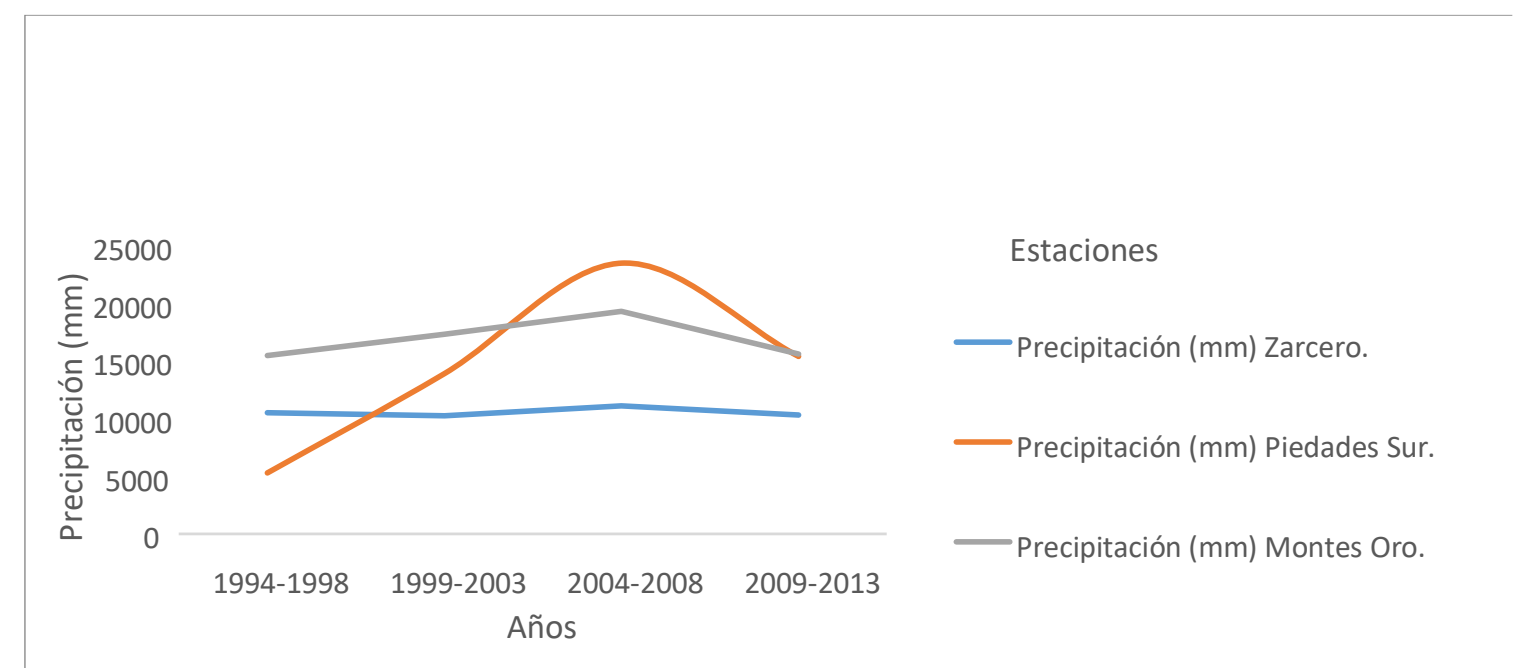

Figura 8: Comparación de la precipitación acumulada cada cinco años para Zarcero, Piedades Sur y Montes de Oro.

Fuente: IMN (2017).

No obstante, las máximas de precipitación tienen lugar cuando se debilitan los vientos alisios del Noreste e incursionan con más intensidad los vientos Oestes Ecuatoriales que ingresan por la costa Pacífica de Costa Rica. En esta temporada es cuando precisamente las Temperaturas superficiales del mar (TSM) son más bajas que el promedio en el océano Pacífico Ecuatorial y más altas que el promedio en el Atlántico Tropical Norte.

En la primera de las figuras se puede observar la poca influencia del anticiclón oceánico sobre el país, o sea existiendo lluvias convectivas. En segunda instancia se produce todo lo contrario, en se da una merma de las precipitaciones, lo que trae consigo subsidencia, con lo cual se reducen las posibilidades de ocurrencia de las precipitaciones. Estos resultados coinciden con lo expuesto por Lapinel et al. (2005).

A su vez, el evento EI Niño-Oscilación del Sur (ENOS) juega un papel importante como elemento de forzamiento de la variabilidad climática natural. Su influencia se refleja 
fundamentalmente en el incremento de los acumulados de precipitaciones durante unos años. En tanto, durante los años El Niño, se produce mermas en la precipitación (Rivero y Ortiz, 2005).

\section{Bibliografia}

Duran, G., Fornet Hernández, E., Hidalgo Mayo A. (2010). Anomalías térmicas positivas y disminución de las lluvias en la comunidad de Guaro Holguín, Cuba. Ciencias Holguín, Revista trimestral, Año XVI, 1-10.

Enfield, D. B., Alfaro, E. J. (1999). Dependence of Caribbean rainfall on the Interaction of the Tropical Atlantic and Pacific Oceans. Journal of Climate, 12, 2093-2103.

Giannnini, A., Kushnir, Y., Cane, M. A. (2000). Interannual Variability of Caribbean rainfall, ENSO and the Atlantic Ocean. Journal of Climate, 13, 297-311.

Hastenrath, Stefan (2002). Variations in Low Latitude Circulation and Extreme Climatic Events in the tropical Americas. Journal of Atmospheric Sciences, 33 (2), 202-215.

Kendall, M. G. (1975). Rank correlation methods. Griffin: London.

Kousky, V. E., Ropelewski, C. F. (1989). Extremes in the Southern Oscillation and their relationship to precipitation anomalies with emphasis on the South American region. Revista Brasileira de Meteorología, 4, 351-363.

Lapinel, B., Centella, A., Gonzáles, I., Fonseca, C., Cutie, V. (2006). Causas de la reciente sequía acaecida en la región oriental de Cuba, Centro del Clima, Instituto de Meteorología, Habana 17, Cuba.

Mann, H. B. (1945). Non-parametric tests against trend. Econométrica, 13, 245-259.

McKee, T. B., Doesken, N. J., Kleist, J. (1993). The relationship of drought frequency and duration to time scales. 8th Conference on Applied Climatology, 179-184.

Moura, A., Shukla, J. (1981). On the Dynamics of Droughts in Northeast Brazil: observations, Theory and Numerical Experiments with a General Circulation Model. Instituto de pesquisas Espaciais, CNPq/INPE, Sao Jose dos Campos, Sao Paulo, Brazil.

Nobre, P., Shukla, J. (1996). Variations of Sea Surface Temperature, Wind Stress, and Rainfall over the Tropical Atlantic and South America. Journal of Climate, 9, 2464-2479. https://doi.org/10.1175/1520-0442(1996)009\%3C2464:VOSSTW\%3E2.0.CO;2.

Quesada, M. E., Waylen, P. R. (2008). Fluctuaciones bimensuales y decadales en la precipitación de San José, Costa Rica. InterSedes: Revista de las Sedes Regionales, vol. IX, núm. 16, 23-34.

Rivero, A., Ortiz, P. (2005). EL índice NAO como elemento modulador de la variabilidad climática del periodo lluvioso en Cuba. Meteorol. Colomb, 9, 67-74.

Trenberth, K. E., Caron, J. M. (2000). The Southern Oscillation Revisited: Sea Level Pressures, surface temperatures and precipitation. Journal of Climate, 13, 4358-4365.

Waylen, P. R., Quesada, M. (2001). The effect of Atlantic and Pacific surface temperatures on the mid-summer drought of Costa Rica. Cuadernos de Investigación Geográfica, $\mathrm{n}^{\circ} .27$, 193-205. http://dialnet.unirioja.es/servlet. 
Zhou, J., Lau, K. M. (2001) Principal Modes of Interannual and Decadal Variability of Summer Rainfall over South America. International Journal of Climatology, 21, 1623-1644. http://dx.doi.org/10.1002/joc.700.

\section{Agradecimientos}

Se agradece a la Vicerrectoría de Investigación, Universidad de Costa Rica por el financiamiento parcial del proyecto 540- B6-021. Este artículo es producto de dicho proyecto de investigación. Un agradecimiento muy especial a los funcionarios del Instituto Meteorológico Nacional encargados del almacenamiento y autorización para el suministro de datos climatológicos.

Artigo recebido em / Received on: 30/07/2019

Artigo aceite para publicação em / Accepted for publication on: 22/10/2019 
Marvin Quesada / Physis Terrae, Vol. 1, n 1, 2019, 107-119

Página intencionalmente deixada em branco 